\title{
Shear-Induced Coalescence in Two-Phase Polymeric Systems. I. Determination from Small-Angle Neutron Scattering Measurements
}

\author{
C. M. ROLAND and G. G. A. BÖHM, The Firestone Tire and Rubber \\ Company, Central Research Laboratories, 1200 Firestone Parkway, Akron, \\ Ohio 44312
}

\section{INTRODUCTION}

The attainment of improved physical properties through the blending of immiscible polymers is a well-established practice. ${ }^{1,2}$ The properties of such multiphase polymer systems reflect to a large extent the nature of the blend morphology, which in turn is influenced by the properties of the constituent materials as well as by the blending conditions employed. In particular, the dispersed-particle size and its distribution are the results of competition between breakdown and coalescence processes. 3,4 Under given mixing conditions an invariant morphology can be attained which represents the steady-state condition existing between particle breakup occurring in the high-shear regions of the mixing vessel and the flow-induced coalescence of these dispersed domains. The larger particles are broken up by the stresses present in the sheared emulsion, while concurrently fluid particles collide and fuse to form larger coalesced species. The dispersion and breakup of fluid particles in a sheared suspension is a much investigated area, ${ }^{3,5-7}$ although one which is as yet not fully understood. Flow-induced coalescence of the dispersed phase and the role it plays in the development of a steady-state morphology has, on the other hand, received considerably less attention.

It is the purpose of this work to quantitatively measure the extent of coalescence occurring during the mixing of rubbery, immiscible polymers, and thereby gain some understanding of its significance in the development of a blend morphology.

\section{EXPERIMENTAL}

For the purposes of this study it was necessary to distinguish coalesced species from the original particles (apart from mere size considerations which reflect particle breakup as well). This was accomplished by preparing compositions containing $5 \mathrm{wt} \%$ polybutadiene (PB) and $95 \mathrm{wt} \%$ polychloroprene $(\mathrm{PC})$ in which the $\mathrm{PB}$ was fully protonated in one case and deuterated in the other. Extensive mixing of these respective precursor samples was carried out in order to establish a steady-state morphology; that is, the dispersed-particle size distribution no longer changes with continued mixing. Upon blending of these two compositions, any loss of the initial isotopic purity of the dispersed phase brought about by shear-induced particle coalescence was then determined from measurements of the magnitude of coherent small-angle neutron scattering. The polybutadienes employed (both deuterated and protonated versions) are listed in Table 
TABLE I

Materials Employed

\begin{tabular}{lcc}
\hline & $\begin{array}{c}\text { Polymerization } \\
\text { index }\end{array}$ & $M_{w} / M_{n}$ \\
\hline Dispersed phase & & \\
PBD-L & 940 & 1.1 \\
PBD-M & 1500 & 1.1 \\
PBD-H & 2800 & 1.1 \\
Continuous phase & & \\
PCP-L & 1100 & 2.8 \\
PCP-M & 1600 & 1.8 \\
PCP-H & 1800 & 4.1 \\
\hline
\end{tabular}

I, along with the polychloroprenes used as a continuous phase. Equal volumes of the isotopically pure $\mathrm{PB} / \mathrm{PC}$ blends were brought together as thin sheets and then passed for varying durations through a 3 -in.-diameter two-roll mill. All milling was done (except where noted) at nominally $80^{\circ} \mathrm{C}$ with a roll separation of $0.010 \mathrm{in}$. The roller speed was $8.5 \mathrm{rpm}$, which corresponds to a surface velocity $U$ equal to $3.4 \mathrm{~cm} / \mathrm{s}$. The milling geometry is depicted in Figure 1. If this flow through the mill is treated as steady Newtonian flow and the velocity through the rollers is assumed to be independent of position along this direction, a simplified hydrodynamic analysis leads to the following expression for the shear rate $^{8}$ :

$$
G_{x y}=3 U_{y}\left(p^{2}-w^{2}\right) / h^{2}\left(1+p^{2}\right)
$$

where $2 h$ represents the slab thickness, which is a function of position along the flow direction, with

$$
p=x / 2 R H_{0}
$$

and

$$
w=X / 2 R H_{0}
$$

Note that at $x=X$, the slab is no longer in contact with the roll surface. The symbols are defined in Figure 1. Both the temperature, as well as $U$, were varied in this study to assess their influence on coalescence. By considering typical conditions employed in our experiments, a shear rate in the nip region of $80 \mathrm{~s}^{-1}$

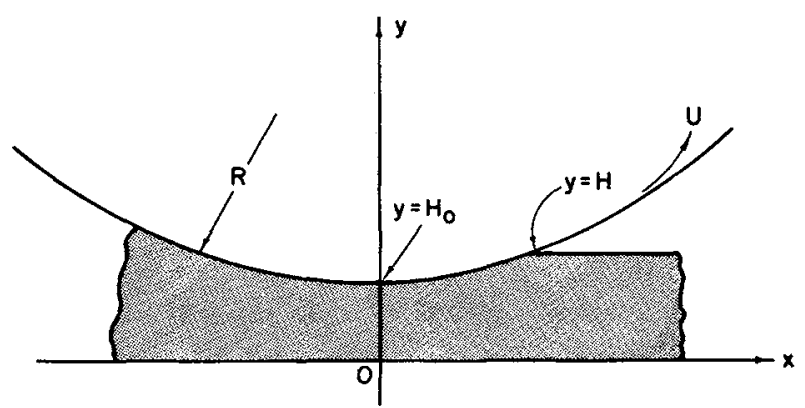

Fig. 1. Illustration of two-roll mill (of radius $R$, nip gap $2 H_{0}$, and roll speed $U$ ) used for polymer mixing. 
can be calculated. The number of collisions per unit time experienced by a spherical particle positioned at $y=y_{1}$ is the product of this local velocity gradient, the number density of particles $N_{T}$, and the cross-sectional area of the particles perpendicular to the flow direction:

$$
\begin{aligned}
J & =\frac{3 N_{T} w^{2} U}{h^{2}} \int_{y_{1}-D}^{y_{1}+D}\left|y^{2}-y_{1}^{2}\right|\left[D^{2}-\left(y-y_{1}\right)^{2}\right]^{1 / 2} d y \\
& =\frac{4 N_{T} w^{2} U}{h^{2}} y_{1} D^{3}
\end{aligned}
$$

Thus the average number of collisions for a particle in the vicinity of the nip per unit time would be

$$
\bar{J}=(0.2 N U / H) D^{3}
$$

where $D$ represents the sum of the radii of the colliding particles. Under the conditions employed, this corresponds to about one collision per second for 400 - $\AA$-radius spheres. If there is a high probability that a collision will lead to coalescence, it can be seen that coalescence of the dispersed PB particles can be very extensive.

In order to ensure that the material contents of a coalesced pair of particles had completely equilibrated, the samples were annealed following shear mixing on the mill. This was carried out at room temperature for approximately $17 \mathrm{~h}$, after which the morphology was stabilized by crosslinking with electron-beam irradiation. A sample was also prepared in which equal volumes of the two isotopic species of $\mathrm{PB}$ were initially preblended in solution and then dispersed into the polychloroprene. This served to simulate the case of an infinite extent of flow-induced coalescence.

Small-angle neutron scattering (SANS) measurements were made on the $30-\mathrm{m}$ SANS instrument at the Oak Ridge National Laboratory. Two sample to detector distances, 18.9 and $6.0 \mathrm{~m}$, were employed in order to expand the accessible range of scattering vectors. The diameters of the source and sample slits were 0.8 and $0.6 \mathrm{~cm}$, respectively, for the longer distance, while $2.0-$ and $1.3-\mathrm{cm}$ slits were used, respectively, for the 6-m measurements. Overlap of the two spectra was then accomplished through calibration with a standard scatterer (an aluminum alloy containing voids). All scattering intensities reported herein are expressed as absolute units $\left(\mathrm{cm}^{-1}\right)$, that is, in terms of the differential scattering cross section for the sample.

\section{BACKGROUND}

\section{Flow-Induced Coalescence}

Coalescence of droplets of a Newtonian fluid dispersed in a second sheared Newtonian fluid occurs through what is essentially a three-step mechanism ${ }^{9,10}$ (Fig. 2). Initially the flow must bring a pair of particles into close proximity. This "collision pair" rotates together in the shear field, remaining separated by a thin film of the continuous-phase fluid. During this rotation, drainage of the intervening film by viscous flow can reduce the film thickness. If the film is reduced to a sufficiently small size that rupture occurs before the pair of particles 

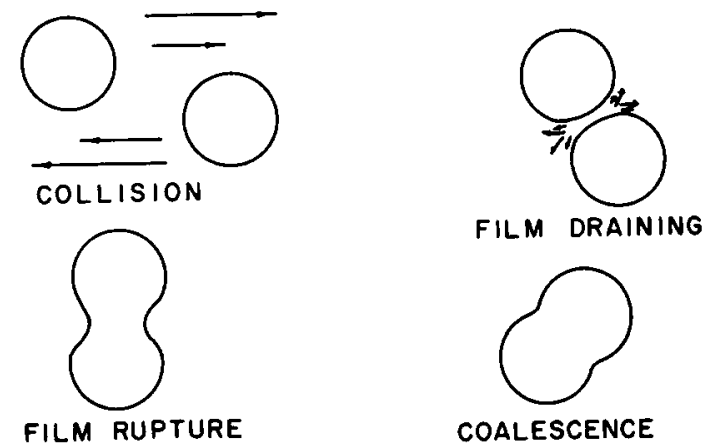

Fig. 2. Idealized depiction of shear-induced coalescence of dispersed Newtonian droplets.

rotates into the next quadrants and is pulled apart by the flow field, then actual coalescence of the drops into a single particle can result. In shearing flows, the contact time for the collision doublet is exceedingly small (on the order of the inverse of the shear rate ${ }^{11}$ ); accordingly, the intervening film has to be rapidly removed so that an actual bond can develop between the particles, enabling them to resist separation in the flow field, and then coalescence will occur.

Although the model described above is based on observations of simple liquid blends, it is nevertheless to be inferred that flow-induced coalescence of polymeric fluid droplets exhibiting non-Newtonian flow behavior will be exceedingly difficult. The initial step, in which the pair of particles is brought together, would be inhibited by elastic recoil of the polymeric matrix. In the second stage of coalescence, the removal of the intervening film would be retarded by the highly viscous polymeric continuous phase. Finally, actual coalescence requires the dispersed droplets to make contact on a microscopic scale and flow together. Figure 3 illustrates the difficulty of this process for high-molecular-weight fluids. It can be observed that a pair of smooth polybutadiene sheets, when plied together under high compressive stress for extended time periods, can still be peeled apart with extensive interfacial failure; that is, even under static conditions, coalescence of rubbery materials is difficult. It might then be expected that in a dynamic situation at typical processing deformation rates, little flow-induced

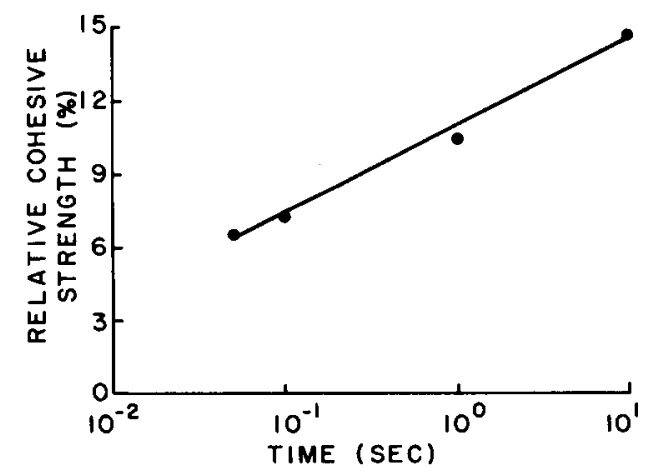

Fig. 3. Peel strength (relative to the bulk cohesive strength) measured for polybutadiene $\left(M_{n}\right.$ $=120,000)$ sheets plied together at ca. $10,000 \mathrm{~Pa}$ for the $\left(80^{\circ} \mathrm{C}\right.$ equivalent $)$ time periods indicated. 
coalescence could occur, given the small time for which two particles remain in close proximity during shear flow (typically less than $1 \mathrm{~s}$ ).

\section{Small-Angle Neutron Scattering}

The small-angle neutron scattering from a heterogeneous blend of polymers will have three contributions-phase scattering resulting from the contrast between the dispersed and continuous domains, molecular scattering from within domains possessing internal contrast by virtue of mixed isotopic species, and finally incoherent scattering. The latter, arising from deviations of scattering length from an average value, is essentially isotropic and thus provides no morphological information (although time-of-flight experiments which measure the energy exchange accompanying incoherent scattering can be used to probe the dynamics of the system). In the experiments conducted by us, the intensity of this incoherent background, while overall quite low, dominates in the far wings of the scattering curve. It was thus possible to determine its contribution and then to subtract it from the measured intensities.

Also present will be molecular scattering from coalesced domains which contain both isotopes of polybutadiene. The magnitude of this contribution when the two isotopic species are randomly dispersed within the particle is just the product of the dispersed-phase number density and the square of the molecular contrast $\Delta b,{ }^{12}$

$$
\frac{d \Sigma}{d \Omega}(Q)=\phi \frac{\chi(1-\chi) \nu \rho}{M}\left(\frac{M}{m}(\Delta b)\right)^{2} \Phi^{2}(Q)
$$

where $M$ and $m$ correspond to the chain and monomer molecular weights, respectively, $\phi$ is the volume fraction of dispersed phase of density $\rho, \chi$ the fraction of PB molecules that are deuterated, and $\nu$ is Avogadro's number. For Gaussian chains of radius of gyration $\tilde{R}$, the form factor is given by ${ }^{13}$

$$
\Phi(Q)=(\sqrt{2} / Q \tilde{R})[Q \tilde{R}-1+\exp (-Q \tilde{R})]^{1 / 2}
$$

It can be seen that for the 5\% PB in PC blends employed, this molecular scattering has a magnitude at $Q=0$ of only about $4 \mathrm{~cm}^{-1}$, which is orders of magnitude less than the phase scattering and thus completely negligible.

The differential cross section for the phase scattering from a heterogeneous blend containing $N_{T}$ dispersed domains per unit volume can be related to an integral over all $N(R)$ domains of volume $V(R)$ ( $R$ represents some linear dimension in the particle), ${ }^{14}$

$$
\frac{d \Sigma}{d \Omega}(Q)=N_{T}\left(\frac{\rho \nu}{m}\right)^{2} K \int_{0}^{\infty} N(R) V^{2}(R) \Phi^{2}(Q R) d R
$$

The form factor for spherical domains of radius $R$ is given by

$$
\Phi(Q R)=3 \sqrt{\pi / 2}\left[J_{3 / 2}(Q R) /(Q R)^{3 / 2}\right]
$$

where $J_{3 / 2}$ represents the Bessel function of order $3 / 2$ and

$$
Q=(4 \pi / \lambda) \sin (\theta / 2)
$$

The thermal neutrons in this work had a wavelength $\lambda$ of $4.75 \AA$ and scattering 
at angles between $0.04^{\circ}$ and $3.7^{\circ}$ was measured $\left(0.003<Q=0.085 \AA^{-1}\right)$.

The contrast factor is related to the difference in the coherent neutron scattering length $b$ for the dispersed and continuous phases, respectively:

$$
K=\left[b_{d}-\left(V_{d} / V_{c}\right) b_{c}\right]^{2}
$$

where each $b$ corresponds to a volume-fraction weighted sum over all monomers in the particle (or phase). In this expression $V_{d}$ and $V_{c}$ refer to the respective molar volumes of dispersed and continuous material. For a polybutadiene particle containing a volume fraction $\chi$ of the deuterated isotope in a polychloroprene matrix, the contrast factor (in barns) is then ${ }^{12}$

$$
K=[6.64 \chi+0.416(1-\chi)-(0.73) 1.748]^{2}
$$

It can be seen that coalescence of a deuterated $(\chi=1)$ PB particle with an equal-sized protonated ( $\chi=0$ ) PB particle will reduce $K$ by

$$
\frac{[6.64(1 / 2)+0.416(1 / 2)-(1.748)]^{2}}{1 / 2\left[(6.664-2.748)^{2}+(0.416-1.748)^{2}\right]}=0.34
$$

The extent of coalescence occurring during the mixing of the blend thus can be determined from the decrease in the coherent small-angle scattering intensity. This intensity is, of course, also influenced by the particle size [eq. (9)]. In order to eliminate this effect so that a direct connection is established between the magnitude of the scattering and the particle's isotopic composition (and thus coalescence), the measured intensities were multiplied by the square of the scattering angle and integrated over all angles. This invariant intensity

$$
\begin{aligned}
\hat{I} & =\int_{0}^{\infty} Q^{2} \frac{d \Sigma}{d \Omega}(Q) d Q \\
& =2 \pi^{2}\left(\frac{\rho \nu}{m}\right)^{2} \sum_{i}\left(\phi_{i} b\left(1-x_{i}\right)+b x_{i}-\frac{V_{d}}{V_{c}} b_{c}\right)^{2}
\end{aligned}
$$

depends only on the volume fraction of coalesced material, which then involves a sum over all types of coalesced particles (i.e., resulting from the coalescence of deuterated and protonated drops of various relative sizes). In practice the integration limit of eq. (15) extends only to scattering angles for which there is measurable intensity in the background-corrected scattering. Inclusion of molecular scattering as described by eq. (8) would lead to a nonconverging integral and thus to an infinite invariant intensity. The phase scattering at high angles, however, makes a negligible contribution to the invariant intensity.

\section{Dispersed-Particle Size Distribution}

In order to relate in a quantitative fashion the SANS intensity to the actual amount of coalescence occurring in the samples, it is necessary to know the probability for a deuterated $\mathrm{PB}$ particle of given size to coalesce with a protonated $\mathrm{PB}$ particle of any particular size. This, in turn, requires information on the size distribution of the dispersed particles. As can be seen in Figure 4, the particles are essentially spherical and exist over a very wide range of sizes. Three approaches were used to assess this size distribution. The most direct is simply to measure the images in electron micrographs. There are problems with this method, all of which tend to result in an underestimation of the true particle size. 


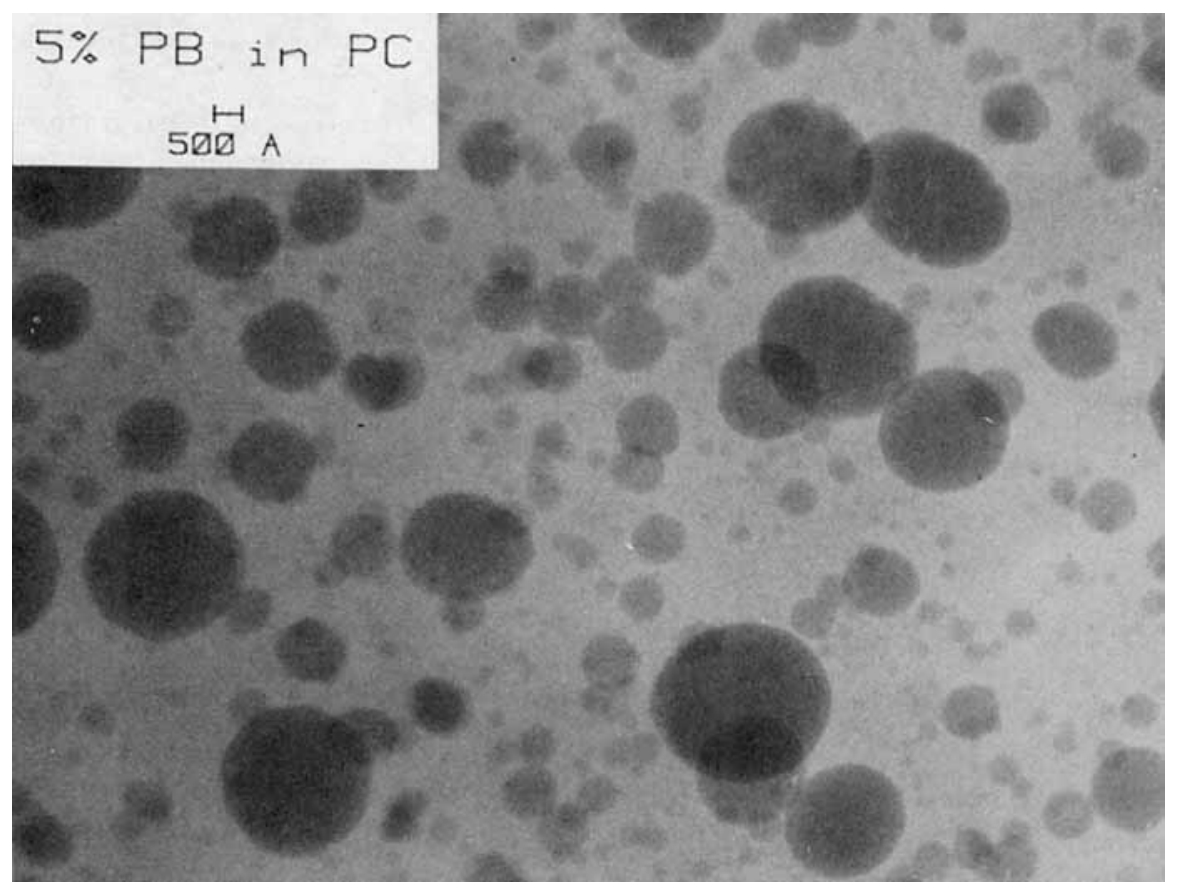

Fig. 4. Transmission electron micrograph of a representative $5 \%$ polybutadiene in polychloroprene blend.

One relates to the difficulty of accurately determining the perimeter of the images. The contrast of the image is inversely proportional to thickness, so that outer regions of an image are less discernible. This problem is accentuated when significant slicing of the particles themselves occurs during ultramicrotoming of a sample. This certainly occurs to some degree with these samples since the average particle diameter (roughly $800 \AA$ ) is very close to the thickness of the microtomed section. In the limit of particle sizes much greater than the section thickness, microtoming through the particles themselves can reduce the apparent average size in the electron micrograph by $11 \% .15$ The results of our analysis of the electron micrograph images are presented in Figure 5 . It can be noted

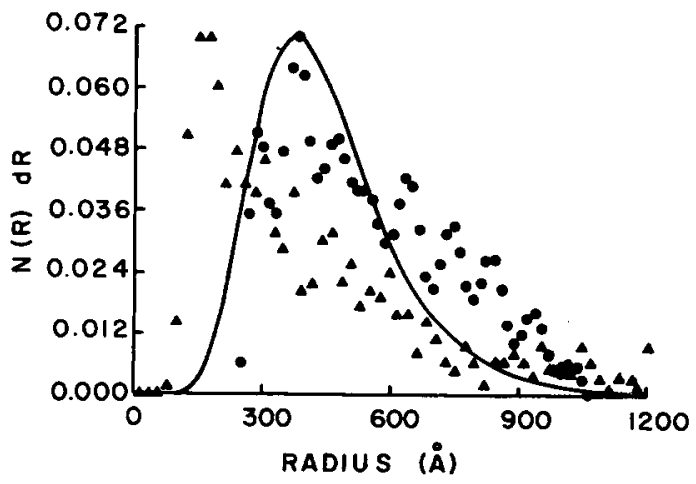

Fig. 5. Particle size distributions as determined by the indicated methods of analysis. 
that, because of the broad size distribution, measurement of about 800 particles gives only moderately good statistics.

A second approach used to evaluate particle size involves an integral transformation of a function directly determinable from the scattering curve measured for a sample containing only one isotope of polybutadiene. For independently scattering spheres, the particle size distribution function (un-normalized) is given by $^{16,17}$

$$
N(R)=R^{-2} \int_{0}^{\infty}\left[Q^{4} I(Q)-C\right] \alpha(2 Q R) d Q
$$

where

$$
\alpha(z)=-z^{2} \frac{d}{d z}\left(z^{-1} \frac{d^{2}}{d z^{2}} \frac{\sin z}{z}\right)
$$

and $C$ represents the Porod constant

$$
C=\lim _{Q \rightarrow \infty}\left[Q^{4} I(Q)\right]
$$

Because the small-angle neutron phase scattering is masked at high angles by the incoherent background, the Porod constant was obtained from small-angle $x$-ray scattering of the same sample. The result of eq. (16) is also displayed in Figure 5. As expected when numerically transforming experimental data, any imprecision in the measured quantities is amplified, and this leads to the fluctuations in $N(R)$ observed in Figure 5. The method is also limited in the range of $N(R)$ actually accessible, in that while the integral of eq. (16) extends over all angles, measurements can only be made over a finite angular range. It is, of course, an inherent problem of using any scattering technique for structural information that only a limited range of reciprocal space is actually probed.

A final evaluation of dispersed-particle size was made by assuming some analytical expression for the distribution, and then using it to calculate scattering curves which were then least-squares fitted to the experimental intensities by variation of the available parameters in the distribution function. Generally, scattering data cannot uniquely determine this distribution. "Scattering equivalent" systems of different morphology can exist, and the shape of the scattering curve is not particularly sensitive to the details of the distribution function. The value in assuming a functional form for the size distribution is that a measure of $N(R)$ is then obtained over all $R$ (although it could be argued that fitting a function to the numerical results of eq. (16) would be perhaps more appropriate if less precise). A couple of distribution functions were employed including

$$
\begin{gathered}
N(R)=R^{-3} \quad(100<R<1000 \AA) \\
N(R)=0 \quad(\text { all other } R)
\end{gathered}
$$

and

$$
N(R)=\frac{1}{R \ln S} \exp \left[-(\ln R-\ln A)^{2} / 2 \ln ^{2} S\right]
$$

The latter log-normal function was actually used in the data analysis. The SANS 


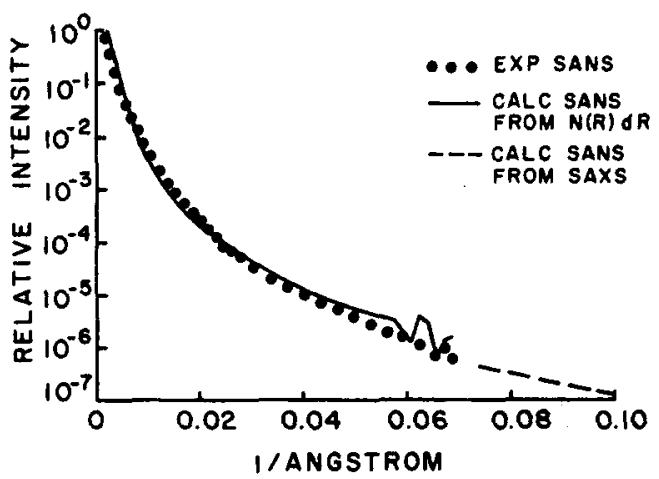

Fig. 6. Experimental (๑) and calculated (-) [according to eqs. (9) and (20)] SANS curves, along with the Porod region determined from small-angle $x$-ray scattering from the same sample (-).

curve employing eq. (20) was calculated according to eq. (9) and the results are shown in Figure 6 along with the experimental measurements. The obtained best-fit values for the mean radius and standard deviation were $425 \AA$ and 0.7 , respectively.

It can be seen that over five decades of scattering intensity the agreement is quite good. The divergence at higher angles reflects both the increased error in experimental measurements and the effect of truncating the calculation (which involves a sum over all particles at each scattering point). The corresponding distribution function is displayed in Figure 5. Given the various sources of error in the three methods of particle size determination, we judge it to be in reasonable agreement with the two other, more direct evaluations of particle size.

\section{RESULTS}

\section{Efficiency of Flow-Induced Coalescence}

Displayed in Figure 7 are the invariant intensities measured for a sample of $5 \%$ PB $\left(M_{n}=80,000\right)$ in PC $\left(M_{n}=144,000\right)$ passed a varying number of times through the mill. Reflecting the effect of flow-induced coalescence, the SANS

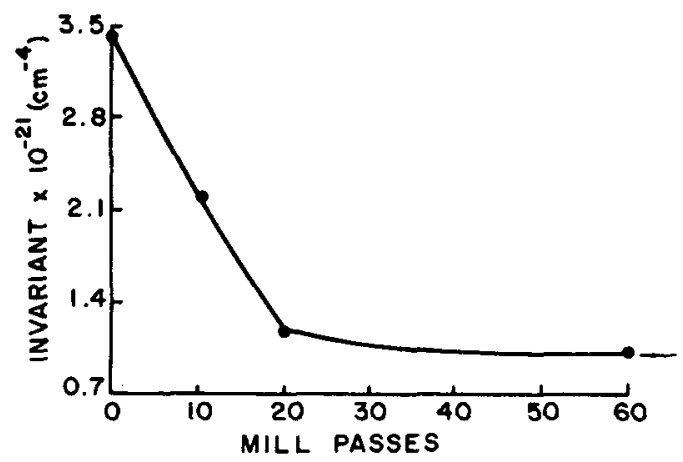

Fig. 7. SANS invariant measured as a function of number of passes through a two-roll mill. The arrow indicates the scattering level for a solution preblended sample corresponding to the equivalent of an infinite mixing time. 
invariant is seen to decrease with time of mixing. The initial point (zero passes) actually is the average invariant intensity measured for a pair of samples containing, respectively, fully deuterated and fully protonated $\mathrm{PB}$ particles. It thus corresponds to no coalescence. A moderate amount of milling (10 passes) reduces the neutron scattering by $36 \%$. An additional 10 passes through the mill causes a proportional reduction in SANS intensity. Continued mixing (up to 60 passes) further reduces the level of neutron scattering, but the rate of decrease is more gradual. This results from the increased frequency of particles, having already undergone coalescence, coalescing again. Lacking their initial isotopic purity, this further coalescence does not alter the scattering contrast very much (the particle composition is approaching an equal concentration of deuterated and protonated molecules). Indicated by the arrow in Figure 7 is the scattering invariant measured for the sample in which equal volumes of the deuterated $P B$ and its protonated analog were premixed in solution and then blended with the polychloroprene. This sample corresponds to an "infinite" extent of coalescence (all particles having an equal level of both the isotopes) and represents, therefore, the lowest value of scattering intensity obtainable. Sixty passes through the mill produces a sample with close to this minimum level of scattering, indicating a very extensive degree of flow-induced coalescence.

It can also be noted that the ratio of the SANS invariant measured for the "solution preblended" sample to the value obtained for the case of no coalescence (zero passes) is 0.30 , very close to the calculated value [eq. (14)], which was based only on the known neutron scattering lengths of the constituent nuclei. This agreement demonstrates the validity of the extrapolation procedure used to obtain the invariant, as well as attesting to the absence of artifacts (e.g., from sample voids or residual PC crystallinity) in the experimental data.

The results suggested by Figure 7 are remarkable in that evidently flow-induced coalescence of rubbery PB particles dispersed in highly viscoelastic PC can be a very efficient process, despite the limited time available for an interacting pair of particles to come together and rapidly form a bond of sufficient strength to resist the repelling forces exerted by the flow field shortly after the "collision" occurs.

By using the log-normal particle size distribution function displayed in Figure 5 , the coalescence probability for a given size sphere could be calculated. It was assumed that the only effect of particle size was its influence on the collision rate [eq. (4)]. Hence, upon collision, the probability that a pair of particles would coalesce was assumed to be independent of their size. Actually, studies of coalescence in simple liquids suggest that smaller particles may coalesce more readily $^{18}$ (presumably owing to easier removal of the intervening film). This is a weak effect, however, and therefore it was assumed here that with respect to size, the coalescence probability is just proportional to the collision rate. By weighting this probability then with the third power of the particle radius, the relation between the volume fraction of dispersed material having undergone coalescence and the magnitude of the SANS invariant was calculated and is displayed in Figure 8. It can be seen that, in agreement with the experimental findings of Figure 7, there is a linear relation between mixing time (and coalescence) and the SANS invariant. Repeated coalescence of individual particles was neglected in this calculation and consequently the curve in Figure 8 does not level off after an extended duration of mixing (as is observed in Fig. 7). The 


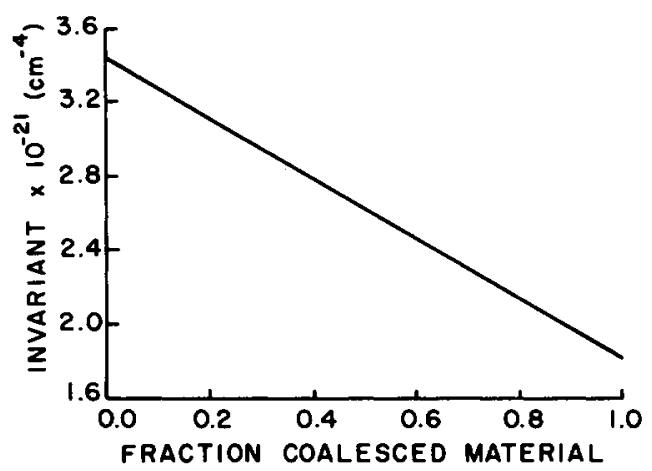

Fig. 8. Computed effect of coalescence on the magnitude of the SANS invariant.

interesting aspect of the data shown in Figures 7 and 8 is the revelation that a moderate amount of mixing (10 passes through the two-roll mill) will induce coalescence in roughly two-thirds of the dispersed phase! It is evident that, notwithstanding the highly viscous nature of these polymeric fluids, flow-induced coalescence does readily occur during the shear mixing of heterogeneous polymers and thus plays a major role in the development of a blend morphology.

\section{Effects of Material Properties and Flow Field on Coalescence}

The inability of a high-molecular-weight continuous phase to prevent coalescence of dispersed domains raises interesting questions concerning the role of the matrix fluid during coalescence. To investigate this aspect, a series of samples were prepared in which the molecular weight of the polychloroprene serving as the continuous phase was varied over the range indicated in Table I, while polybutadiene of $M_{n}=51,000$ served as the dispersed phase. The test samples were mill mixed as before. SANS invariants measured on these blends are listed in Table II. One notes that a viscosity increase from 5160 to $7250 \mathrm{P}$ (measured at the nominal mixing shear rate and temperature) causes the extent of coalescence to be reduced by approximately $60 \%$ (based on $\hat{I}$ and the data of Fig. 8). Thus the nature of the continuous phase has an effect on the rate of coalescence. However, it appears unlikely that removal of the highly viscous film separating a pair of coalescing particles can occur through viscous flow. More likely it involves a tearing process in which the thin film is strained and subsequently fractured. Although an inverse relation between the extent of coalescence occurring and the polychloroprene tear strength (measured in a

TABLE II

Matrix Effects (Dispersed Phase PBD-L)

\begin{tabular}{lcccc} 
& Viscosity & Cohesive strength & \multicolumn{1}{c}{$I \times 10^{-21}\left(\mathrm{~cm}^{-4}\right)$} \\
& $\left(\mathrm{P} ; 80^{\circ} \mathrm{C}, 80 \mathrm{~Hz}\right)$ & $\mathrm{kJ} / \mathrm{m}^{3}\left(G \simeq 3 \mathrm{~s}^{-\mathbf{1}}\right)$ & $10 \times$ & $20 \times$ \\
\hline PCP-L & 5160 & 200 & 1.84 & 0.92 \\
PCP-M & 5760 & 660 & 2.18 & 1.18 \\
PCP-H & 7250 & 1080 & 2.49 & 1.31 \\
PBD-L/PCP-M & $\cdots$ & 20 & $\cdots$ & $\cdots$ \\
\hline
\end{tabular}


TABLE III

Influence of Dispersed Phase (Matrix PCP-M)

\begin{tabular}{lccc} 
& \multicolumn{2}{c}{$I \times 10^{-21}\left(\mathrm{~cm}^{-4}\right)$} & \\
\cline { 2 - 4 } & $10 \times$ & $20 \times$ & $G \eta J(t)$ \\
\hline PBD-L & 2.18 & 1.18 & 5.82 \\
PBD-M & 2.14 & 1.13 & 2.03 \\
PBD-H & 3.01 & 1.46 & 0.88 \\
& Collision doublet lifetime $4 \pi / G \simeq 0.2 \mathrm{~s}$ & \\
\hline
\end{tabular}

peeling geometry at an arbitrary strain rate) does exist (Table II), the detailed nature of the film removal mechanism is actually unknown. Since the maximum adhesive strength developed between the $\mathrm{PB}$ dispersed phase and the $\mathrm{PC}$ was found to be at least an order of magnitude weaker than the matrix cohesive strength, most film removal is undoubtedly via slippage at the drop interface once fracture has occurred.

Since removal of the intervening film alone is not sufficient to effect coalescence of viscous drops, a series of measurements were made to determine the influence of the rheology of the dispersed phase on the coalescence efficiency. As may be inferred from the data in Figure 3, shear-induced coalescence of high-molecular-weight rubbery particles embedded in a liquid matrix is in fact contrary to expectations. The ability of entangled molecular fluids to coalesce in the vanishingly small time period over which a pair of drops interact $(<1 \mathrm{~s})$ would not be predicted from observation of the fusion of macroscopic sheets (which does not model the flow-induced coalescence process, but likewise entails macromolecular diffusion across an interface).

To attempt to shed further light on this, a series of samples were prepared in which the polybutadiene serving as the dispersed phase (again present initially in equal amounts of the two isotopes), had a number-average molecular weight ranging from 51,000 to 152,000 (Table I). The resulting SANS invariants measured for these blends are listed in Table III. It can be seen that an increase of $M_{n}$ from 51,000 to 81,000 had no measurable effect on coalescence (as evidenced by the equal scattered intensities). A further increase of $M_{n}$ to 152,000, however, severely inhibited coalescence (by about a factor of 3 according to the data of Fig. 8). In the absence of any detailed knowledge of the interaction mechanism of a pair of viscoelastic droplets suspended in a sheared viscoelastic medium, interpretation of these results can only be speculative. In the flowinduced coalescence of simple fluids it has been observed that upon approach the particles flatten out, thereby producing a greater contact area. ${ }^{10}$ As the collision doublet rotates and begins to be pulled apart by the shear field, any bond developed between the particles must be able to resist this separating action of the bulk flow. While incipient coalesced species of higher molecular weight could better resist fracture by the flow field, the need for the particles to flatten out and make intimate contact on a microscopic scale would argue for enhanced coalescence of more deformable particles. Moreover, actual bonding of the fluid droplets would also require interdiffusion and reentangling of the polymer chains. Recent investigations ${ }^{19}$ have shown that, notwithstanding the large self-diffusion rates typically observed for macromolecules [e.g., $10^{-13} \mathrm{~cm}^{2} / \mathrm{s}$ (ref. 20)], this interdiffusion in polybutadiene elastomers does not necessarily lead to complete 
TABLE IV

Effect of Flow Field (PBD-L in PCP-M)

\begin{tabular}{cccc}
\hline$G\left(\mathrm{~s}^{-1}\right)$ & Passes & Temperature & $\hat{I} \times 10^{-21}\left(\mathrm{~cm}^{-4}\right)$ \\
\hline 80 & 60 & $80^{\circ} \mathrm{C}$ & 1.03 \\
80 & 60 & $\mathrm{RT}$ & 2.56 \\
\hline
\end{tabular}

disappearance of the interface between contacting PB specimens in the expected time scale of a second or less.

As a measure of the deformability of the various polybutadienes employed in this study, their creep compliance was measured (by time-temperature superposition) at the (order of magnitude) time available for drops to coalesce when sheared at $80 \mathrm{~s}^{-1}$ (approximately $4 \pi / G \simeq 0.2 \mathrm{~s}$ ). The product of this compliance and the stress developed in polychloroprene when sheared at $80 \mathrm{~s}^{-1}$ (measured by capillary rheometry) is listed in Table III. It is interesting that a large change in droplet deformability has no measurable effect on coalescence, while a further stiffening of the dispersed-phase material results in strong inhibition of coalescence. Undoubtedly the competing effects alluded to above (bond formation versus bond breakage) are in some part responsible for this seemingly anomalous behavior. Moreover, it has to be emphasized that the manner in which viscoelastic droplets interact when dispersed in a sheared viscoelastic medium is unknown; indeed, even in the case of simple fluid blends, the particles do not actually follow the streamlines, but rather interact in a complex fashion. ${ }^{21,22}$ The value of data such as those displayed in Table III is that they can provide some insight into the nature of the coalescence mechanism. Presumably at a slower deformation the highest-molecular-weight PB would more easily coalesce and, by the same token, the behavior of the lower-molecular-weight PBs would diverge at an increased shear rate.

In order to elucidate the role of flow-field properties in inducing coalescence, the temperature and deformation rate during mixing were varied, and SANS measurements were then made on the resulting samples. By reducing the nominal mixing temperature to room temperature, severe inhibition of coalescence was observed (Table IV). This is in accord with the previously discussed data in Table II, in which it was found that a more viscous (or cohesive) matrix retarded coalescence. Essentially the same effect is of course obtained by a temperature reduction. In addition, at room temperature a significant amount of "plug flow" of the viscous polychloroprene was observed which reduces the incidence of shear-induced collisions and so too coalescence.

A more interesting parameter to vary is the deformation rate. This can be accomplished by changing the roller speed or separation, although the latter could also significantly alter the nature of the flow field. As the data in Table $\mathrm{V}$ reveal, increasing the average shear rate by a factor of 2 (by doubling the roller speed) resulted in an increase in the extent of coalescence (for a fixed number of mill passes). If the limiting factor for a colliding pair of particles to coalesce is the rapid removal of the intervening film, certainly a higher shear rate would be expected to reduce the coalescence efficiency. Indeed, observations on sheared Newtonian liquid droplets have shown that to be the case. ${ }^{23}$ The apparent discrepancy seen in this study could be a result of the viscoelastic nature of these fluids. The rupture of the intervening film, for example, can be accomplished 
TABLE V

Effect of Flow Field (PBD-L in PCP-M)

\begin{tabular}{rccc}
\hline$G\left(\mathrm{~s}^{-1}\right)$ & Passes & Temperature $\left({ }^{\circ} \mathrm{C}\right)$ & $I \times 10^{-21}\left(\mathrm{~cm}^{-4}\right)$ \\
\hline 80 & 10 & 80 & 2.18 \\
80 & 20 & 80 & 1.18 \\
160 & 10 & 80 & 1.92 \\
160 & 20 & 80 & 1.04 \\
\hline
\end{tabular}

at lower strain when a higher shear rate is employed. The discontinuous nature of the mixing procedure could also produce more coalescence at higher shear rates due to a resulting faster relaxation yielding more nearly spherical particles prior to the next pass; the resulting larger cross-sectional area would then lead to enhanced collision rates.

\section{SUMMARY}

It has been found that flow-induced coalescence occurs at a very rapid rate during the mixing of polymeric fluids. Furthermore, the rheological properties of the dispersed and continuous phases, as well as the nature of the flow field used in their blending, can greatly influence this coalescence. The significance of these findings is that in the development of a mixing scheme to obtain a desired morphology with minimum expenditure of time or energy, attention is usually focused only on the particle breakup aspects of the blending process. The competing coalescence can, however, be accelerated by the same conditions often employed to facilitate particle breakup (e.g., higher shear rates, reduced dispersed-phase viscosity).

A better understanding of the mechanism for coalescence of viscoelastic fluids is clearly required. In this manner optimal blending of immiscible polymers can be achieved with respect to both the nature of the final material and the ease with which it is obtained.

The authors wish to express their appreciation to Dr. D. P. Tate and Dr. T. W. Bethea of Firestone Central Research, who synthesized the various polybutadienes from butadiene gas, and to Dr. G. D. Wignall of Oak Ridge National Laboratory for helpful suggestions. We also thank the Firestone Tire and Rubber Company for permission to publish this work.

\section{References}

1. R. D. Deanin, A. A. Deanin, and T. Sjoblom, in Polymer Science and Technology, Vol. 4, L. H. Sperling, Ed., Plenum, New York, 1974, p. 63. 1976.

2. J. A. Manson and L. H. Sperling, Polymer Blends and Composites, Plenum, New York,

3. C. J. Nelson, G. N. Avgeropoulos, F. C. Weissert, and G. G. A. Böhm, Angew. Makromol. Chem., 60/61, 49 (1977). 1980 .

4. G. G. A. Böhm, presented at Conference on Engineering Science Foundation, Asilomar, Calif.,

5. H. P. Grace, presented at 3rd Engineering Foundation Research Conference on Mixing, Andover, NH, 1971.

6. H. B. Chin and C. D. Han, J. Rheol., 24, 1 (1980).

7. R. W. Flumerfelt, Ind. Eng. Chem. Fundam., 11, 312 (1972).

8. J. M. McKelvy, Polymer Processing, Wiley, New York, 1962, Chap. 9. 
9. E. Barnea and J. Mizrahi, Trans. Inst. Chem. Eng., 53, 75 (1975).

10. R. S. Allen and S. G. Mason, J. Colloid Sci., 17, 383 (1962).

11. W. Bartok and S. G. Mason, J. Colloid Sci., 14, 13 (1959).

12. S. N. Jahshan and G. C. Summerfield, J. Polym. Sci. Polym. Phys. Ed., 18, 1859 (1980).

13. P. J. Flory, Statistical Mechanics of Chain Molecules, Interscience, New York, 1969, Chap. 9.

14. A. Guinier and G. Fournet, Small-Angle Scattering of X-Rays, Wiley, New York, 1955.

15. E. R. Weibel, in Principles and Techniques of Electron Microscopy, Vol. 3, M. A. Hoyat, Ed., Van Nostrand, New York, 1973, Chap. 6.

16. J. H. Letcher and P. W. Schmidt, J. Appl. Phys., 37, 649 (1966).

17. O. L. Brill and P. W. Schmidt, J. Appl. Phys., 39, 2274 (1968).

18. G. E. Charles and S. G. Mason, J. Colloid Sci., 15, 206 (1960).

19. C. M. Roland, unpublished.

20. J. D. Skewis, Rubber Chem. Technol., 39, 217 (1966).

21. G. R. Zeichner and W. R. Showalter, AIChE J., 23, 243 (1977).

22. C. J. Lin, K. J. Lee, and N. F. Sather, J. Fluid Mech., 43, 35 (1970).

23. W. Bartok and S. G. Mason, J. Colloid Sci., 14, 13 (1959).

Received March 31, 1983

Accepted August 12, 1983 